\title{
PROCESSO DE BIORREMEDIAÇÃO USANDO O FERTILIZANTE NPK, EM EVENTUAIS DERRAMES DE ÓLEO - EXPERIMENTOS LABORATORIAIS
}

\author{
ANABELA PORTO ROSA ${ }^{1} \&$ JORGE ALBERTO TRIGÜIS ${ }^{2}$
}

\begin{abstract}
Resumo Biorremediação é uma técnica que utiliza microorganismos para reduzir a concentração e/ou a toxicidade dos poluentes, podendo ser promissora na limpeza de derrames de óleo em ambientes costeiros. O objetivo desse trabalho foi analisar as variações, em testes de laboratório, da biorremediação usando o fertilizante NPK, visando acelerar a biodegradação de derrames de óleo. O processo de biorremediação foi monitorado através de análises geoquímicas das amostras de óleo extraídas dos sedimentos. A água do mar usada durante o processo foi analisada quanto ao teor de nutrientes (íons fosfato e amônia) e realizados testes de toxicidade (testes de Microtox). Os resultados mostraram que o fertilizante NPK pode ser usado na biorremediação em ambientes costeiros contaminados por óleo.
\end{abstract}

Palavras chaves: Bioremediação, biodegradação, fertilizante NPK

\begin{abstract}
BIOREMEDIATION USING NPK FERTILIZER ON SPILL OIL - LABORATORY EXPERIMENTS Bioremediation is a technique that uses microorganisms to reduce the concentration and/or the toxicidade of the pollutants, so it can be considered as an environmentally promising alternative to clean oil spill shorelines. The main objective of this work was to analyze the behavior of laboratory tests on biorremediation, with NPK nutrient to stimulate biodegradation of oil spills. The bioremediation process was monitored by geochemical analysis of the extracted oil from the sediments. The seawater used on the process was analyzed for nutrients contents (phosphate and ammonium ions) and also for toxicity (Microtox tests). All results show that the NKP fertilizer can be used on bioremediation on sites affected by oil spill.
\end{abstract}

keywords: Bioremediação, biodegradação, fertilizante NPK.

INTRODUÇ̃̃O A grande utilização do ambiente marinho pela indústria petrolífera faz deste produto e de seus derivados os principais poluentes em regiões oceânicas, podendo atingir áreas litorâneas e ameaçar ecossitemas sensíveis, interferindo diretamente nas atividades econômicas como a pesca e o turismo (Mitchell et al. 1993). Ao ocorrer um derrame, os processos intempéricos que atuam sobre o óleo, alterando suas propriedades físicas e químicas são: biodegradação, espalhamento, evaporação, dissolução, dispersão, emulsificação, sedimentação e foto-oxidação (Prince 1993).

Biodegradação ocorre através da ação de microrganismos, como bactérias e fungos, presentes no ambiente (Atlas 1981, Alexander 1981, Crapez 1982, Slivins \& Tremier 1985). Entretanto, esse processo pode estar limitado à disponibilidade de nutrientes, composição e abundância das comunidades microbianas, salinidade, temperatura, concentração de oxigênio dissolvido e distribuição e natureza do óleo derramado. Biorremediação é a aceleração deste processo, através da adição de microrganismos específicos (bioaumentação), nutrientes e/ou outras substâncias que acelerem a reprodução de tais microrganismos (bioestimulação), catalisando a reação no meio ambiente contaminado (Rytkonen et al. 1997).

OBJETIVOS O presente trabalho tem como objetivo principal testar a técnica da biorremediação, em simulações de laboratório, derramando óleo em sedimentos costeiros e aplicando o nutriente NPK para estimular a biodegradação. Através dos dados obtidos ao longo dos experimentos, pretende-se avaliar a aplicabilidade da técnica de biorremediação na mitigação de impactos ambientais, devido a eventuais derrames de óleo nesses ambientes.

A simulação experimental é efetuada no período de um mês e seu monitoramento inclui: análises químicas do óleo, análises quantitativas da população microbiana, análises da toxicidade e das concentrações de nutrientes na água usada nos experimentos.

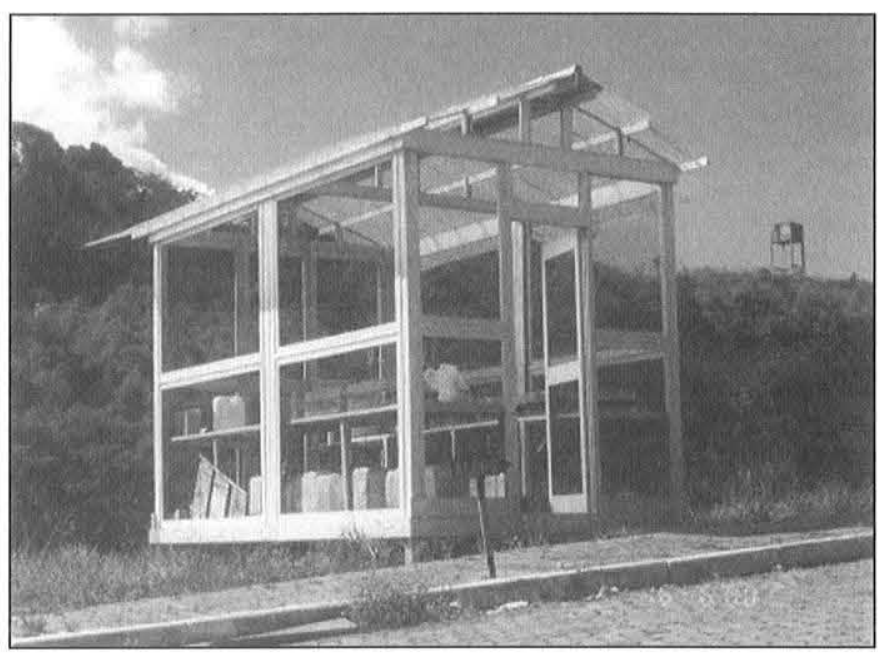

Figura 1 - Galpão usado da simulação de derrames de óleo, nas instalações do LENEP/Macaé-RJ.

UNIDADES DE SIMULAÇÃO Com a finalidade de realizar os experimentos de simulação dos derrames de óleo nos sedimentos, construiu-se um galpão de $15 \mathrm{~m}^{2}$, na parte externa do laboratório de geoquímica. Este possui cobertura com telhas transparentes e paredes laterais revestidas de tela, permitindo a entrada de ar e luz natural, sob condições de temperatura ambiente (Figura 1).

Para simular o derrame de óleo em sedimentos costeiros são utilizadas doze provetas de vidro com capacidade de $2 \mathrm{~L}, 50 \mathrm{~cm}$ de altura e $8 \mathrm{~cm}$ de diâmetro. Para a simulação das marés, na base de cada coluna são adaptadas torneiras de vidro usadas no controle da entrada e saída de água ao longo da simulação. Em 


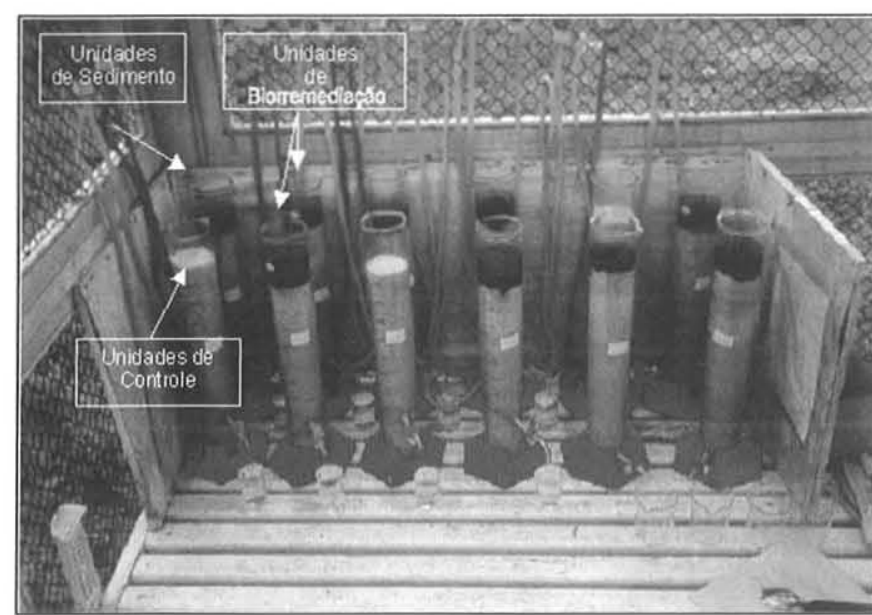

Figura 2 - Distribuição das Unidades de Simulação ao longo do experimento.

cada torneira, é acoplado um reservatório de água, através de um sistema de vasos comunicantes, responsáveis pela entrada de água no sistema.

Os sedimentos são transferidos para as provetas de vidro e após a transferência, é introduzido $500 \mathrm{ml}$ de água do mar em cada coluna, até o topo dos sedimentos, referente à simulação da maré alta, efetuada duas vezes ao dia, em intervalos de 12 horas.

Aprisiona-se a água do mar nas colunas e, posteriormente se adiciona $16 \mathrm{ml}$ de óleo em cada coluna. O volume de óleo empregado nesta simulação respeita os valores encontrados na bibliografia de $2,8 \mathrm{~kg} / \mathrm{m}^{2}$ (Rytkonen et al. 1997), ou seja, $16 \mathrm{ml}$ de óleo com densidade $=0,89$ corresponde a $14 \mathrm{~g}$ de óleo.

Montam-se unidades de simulação distintas, e cada coluna possui uma duplicata com objetivo de se testar a reprodutibilidade do experimento (Fig. 2):

- Unidades de Sedimentos - colunas contendo apenas sedimentos;

- Unidades de Controle - unidades que contém sedimentos e óleo;

- Unidades de Biorremediação - colunas contendo sedimento, óleo e nutrientes. A quantidade de nutriente aplicado em cada coluna é de 1,5 g de NPK, em intervalos de dez dias, constituindo três aplicações ao longo da simulação (um mês). A quantidade total de fertilizante aplicado perfaz 30\% do volume de óleo derramado.

Coleta de Sedimentos Os sedimentos usados na simulação são coletados na praia das Dunas, no município de Cabo Frio/ RJ. Os sedimentos são amostrados em tubos de PVC, com 7,5 $\mathrm{cm}$ de diâmetro e $50 \mathrm{~cm}$ de altura, os quais são enterrados na região de intermaré, ao longo da praia (Fig. 3). Posteriormente, os tubos contendo os sedimentos são retirados, lacrados e enviados ao laboratório.

A morfologia da maioria dos grãos é achatada, com poucos exemplares alongados e eqüantes. Mostram baixa esfericidade, variando de arredondados a subarredondados, com textura superficial polida, provavelmente decorrente do agente transportador. Quanto à mineralogia, observa-se um predomínio de grãos de quartzo límpidos, com a presença de alguns minerais opacos em pequenas proporções, provavelmente constituídos de minerais pesados.
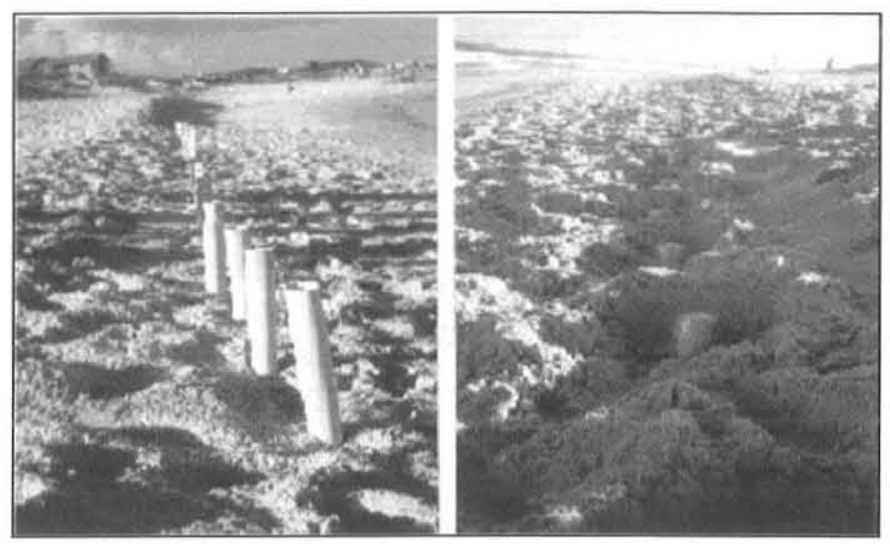

Figura 3 - Coleta de sedimentos na Praia das Dunas, municipio de Cabo Frio/RJ. (A) Disposição das PVC ao longo do perfil praial; (B) Coleta do material sedimentar até a profundidade de $50 \mathrm{~cm}$.

Através da análise estatística dos resultados obtidos, calculase o tamanho médio dos grãos usando a fórmula (Teixeira Neto 1980): Média $=\Sigma$ (intervalo $x$ freqüência $) / 100$ e o resultado em torno de $0,33 \mathrm{~mm}$, refere-se à fração de Areia Média.

Para o cálculo do desvio padrão utiliza-se a fórmula: Desvio Padrão $=\sqrt{ }\left[\Sigma(\text { Média }- \text { intervalo })^{2} / 100\right]$ e obteve-se um resultado em torno de 0,59 o qual caracteriza a seleção dos sedimentos como boa.

Coleta da Água do Mar A água do mar usada durante a simulação é coletada na Praia da Tartaruga, no município de Rio das Ostras/RJ, em intervalos de 48 horas. Após a coleta, a água é armazenada em um recipiente plástico com capacidade de 20 L, e enviada ao laboratório para ser usada durante a simulação de derrame.

Diariamente, efetuam-se medidas do $\mathrm{pH}$ das águas coletadas na praia, antes e durante a as simulações. Os valores mantêm-se constantes em torno de oito, não sendo observada nenhuma variação desse valor ao longo dos experimentos. Essa condição de estabilidade do $\mathrm{pH}$ pode ser uma condição favorável ao desenvolvimento de bactérias heterotróficas presentes no ambiente (Bartha 1996 apud Del'Arco 1999).

Os valores das temperaturas obtidos ao longo do experimento mostram valores elevados, típicos do verão em regiões de climas tropicais. São medidas as temperaturas mínimas e máximas do ambiente, com valores em torno de 23 e $34^{\circ} \mathrm{C}$, respectivamente. Esse intervalo de temperatura propicia o desenvolvimento da população microbiana encontrada nas unidades de simulação (Cookson 1995).

Óleo Bruto Neste trabalho utiliza-se um óleo leve produzido na Bacia de Campos, do Campo de Albacora, cedido pelo Laboratório de Fluídos da unidade operacional da PETROBRAS/ Bacia de Campos (GELAF/PETROBRAS/BC). Esse óleo possui as seguintes propriedades: ${ }^{\circ} \mathrm{API}=26,9$, densidade $=0.8892$ $\mathrm{g} / \mathrm{P}$, viscosidade dinâmica $=54,2 \mathrm{cp}$.

Análises de cromatografia líquida do óleo determinam os percentuais das frações de hidrocarbonetos saturados, aromáticos e compostos NSO contidos no mesmo (Fig. 4).

A escolha de um óleo leve provém do fato do mesmo ser mais facilmente biodegradado pela comunidade microbiana, em função de ser constituído por uma percentagem maior de hidrocarbonetos saturados (mais que 50\%) (Tissot \& Welte 1978).

O cromatograma obtido pela análise da fração dos hidrocarbonetos saturados (alifáticos), mostra a distribuição e abun- 


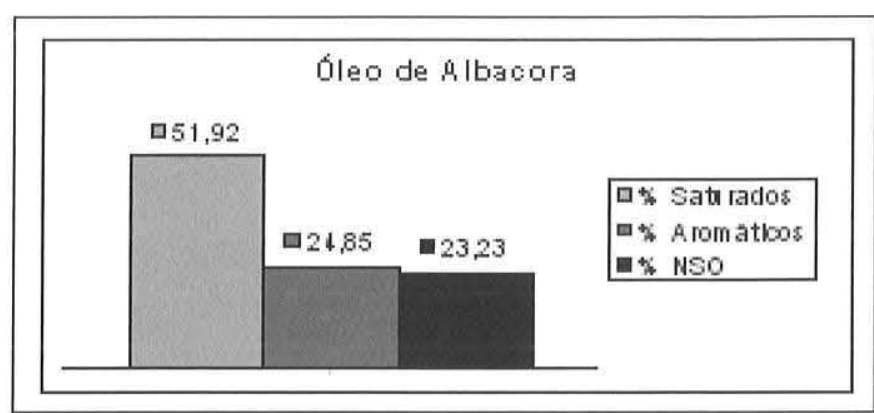

Figura 4 - Fracionamento do óleo Albacora por cromatografia liquida.

dância relativa dos $n$-alcanos e isoprenóides, onde se observa o predomínio dos hidrocarbonetos leves, comprovando a não atuação do processo de evaporação (Fig.5). A ausência da mistura complexa não resolvida (UCM), a razão pristano/fitano $<2$ e os altos valores obtidos das razões $n-\mathrm{C}_{17} /$ pristano e $n-\mathrm{C}_{18} /$ fitano caracterizam o óleo como não biodegradado (Tissot \& Welte 1978, Peters \& Moldowan 1993, Wang et al. 1999).

$\mathrm{Na}$ análise da fração dos hidrocarbonetos saturados, pela cromatografia gasosa acoplada a espectrometria de massa, são obtidos fragmentogramas mostrados na Fig.6. Analisam-se os biomarcadores cíclicos mais suscetíveis a biodegradação, dentre os quais destacam-se: terpanos (fragmentogramas m/z 191) e esteranos (fragmentogramas m/z 217).

Nos fragmentogramas m/z 191, o óleo original apresenta: terpanos tricíclicos com 19 a 23 átomos de carbono; terpanos tetracíclicos contendo de 24 a 28 átomos de carbono; e terpanos pentacíclicos ou hopanos variando de 28 a 35 átomos de carbono. Já nos fragmentogramas $\mathrm{m} / \mathrm{z} 217$, são encontrados diasteranos e esteranos, onde os primeiros mostram-se mais resistentes a biodegradação em função da sua complexidade estrutural (Seifert \& Moldowan 1979, Connan 1984).

Nutriente Com base na bibliografia (Sveum et al. 1991, Merlin et al. 1994, Al-Hadhrami et al. 1997) é utilizado o fertilizante NPK como agente estimulador da biorremediação. Esse produto contém fosfato de amônia $\left[\left(\mathrm{NH}_{4}\right)_{3} \mathrm{PO}_{4}\right]$, sulfato de amônia $\left[\left(\mathrm{NH}_{4}\right)_{2} \mathrm{SO}_{4}\right]$ e cloreto de potássio [KCl] nas proporções $(\mathrm{N}: \mathrm{P}$ : K) de 10:10:10. Trata-se de um fertilizante de plantas, facilmente encontrados no mercado a preços reduzidos.

\section{METODOLOGIA}

Cromatografia Líquida à Média Pressão (CLMP) A cromatografia é o método físico-químico de separação de componentes de uma mistura e depende da distribuição dos constituintes de duas fases imiscíveis (estacionária e móvel). O objetivo da cromatografia líquida à média pressão (CLMP) é o fracionamento da amostra de óleo em grupos de hidrocarbonetos saturados, hidrocarbonetos aromáticos e compostos polares (Chhatre et al. 1996).

O sistema CLMP separa os componentes usando, como fase estacionária, a combinação de sílica termicamente desativada e $n$-hexano como eluente, para obtenção de frações bem definidas e com alto grau de reprodutibilidade. Esse sistema é constituído por: uma bomba para fornecimento da fase líquida; um módulo para injeção de até 15 amostras; um detector ultravioleta (UV); um detector de índice de refração (IR); um coletor automático das frações; e um controlador.

Cromatografia Gasosa com Detector Ionizado por Chamas (CG/FID) O cromatógrafo utilizado é um Hewlett Packard (HP), modelo 6890, com injetor automático do tipo split/spli-

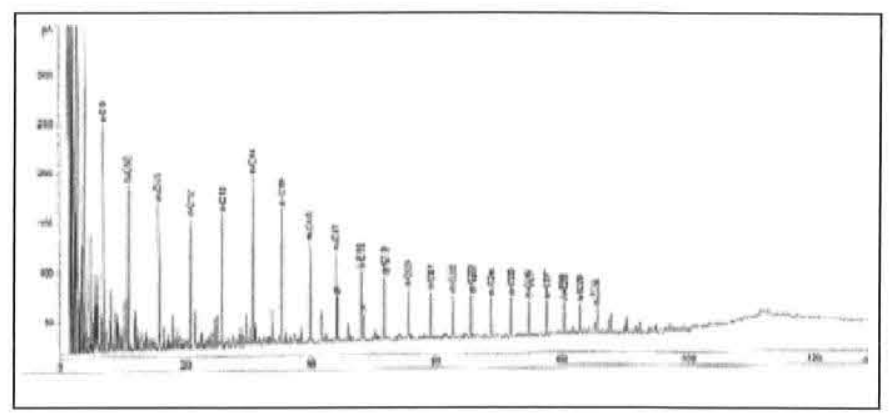

Figura 5 - Cromatograma dos hidrocarbonetos saturados do óleo de Albacora.

tless e um detector de ionização por chama (CG/FID).

É utilizada uma coluna capilar de sílica fundida, marca J \& W Scientifc DB-5, com fase estacionária fenilmetilsiloxano, de baixa polaridade. A coluna contém $30 \mathrm{~m}$ de comprimento, 0,25 $\mathrm{mm}$ de diâmetro interno e $0,25 \mu \mathrm{m}$ de espessura de filme, suportando temperatura máxima de $350^{\circ} \mathrm{C}$. A programação utilizada nas análises cromatográficas é: volume de amostra injetada $=$ $1 \mu \mathrm{l}$; temperatura do injetor $=280^{\circ} \mathrm{C}$; temperatura do detector $=$ $340^{\circ} \mathrm{C}$; programa do forno $=40$ a $320^{\circ} \mathrm{C}$, a $2,5^{\circ} \mathrm{C} / \mathrm{min}$; período isotérmico final $=18 \mathrm{~min}$, a $320^{\circ} \mathrm{C}$; gás carreador $=$ hélio, com fluxo de $2,2 \mathrm{ml} / \mathrm{min}$.

\section{Cromatografia Gasosa Acoplada ao Espectrômetro de Mas-} sas (CG/EM) As condições do cromatógrafo são às mesmas citadas no item anterior. Também são utilizado o espectrômetro de massa marca Hewlett Packard (HP), modelo 5972 MSD.

Quanto às condições analíticas do espectrômetro de massa, podemos citar: temperatura da interface $=285^{\circ} \mathrm{C}$; ionização $=$ por impacto de elétrons; energia do feixe de elétrons $=70 \mathrm{eV}$; varredura $=$ por monitoramento seletivo de íons.

Contagem Microbiana A análise microbiana é realizada conforme método de contagem por plaqueamento. Na contagem das bactérias heterotróficas emprega-se cultura sólida, conhecida como Tripli Soil Agar (TSA), que serve de fonte de energia. Entretanto, na contagem do número de bactérias degradadoras (hidrocarbonoclásticas) utiliza-se cultura líquida, conhecida como Bushwell Haas (BH) e nessa análise, o meio de cultura não serve como fonte de energia para as bactérias, sendo necessário adicionar óleo árabe leve. A análise microbiana é efetuada visualmente, constando-se o número de colônias obtidas por grama de sedimento (CFU/g). Teoricamente, cada colônia é proveniente de um simples organismo e a contagem destas colônias é multiplicada pelo fator de diluição de cada amostra, obtendo-se assim o número de células totais (Pelczer et al. 1980).

Análise de Toxicidade Para a análise da toxicidade das amostras de água são usadas bactérias marinhas luminescentes (Photobacterium Phosphoreum), as quais emitem luz como parte do seu processo metabólico. A medida dessa luminescência é feita, sob condições específica através do dispositivo fotodetector padrão, denominado analisador Microtox (Ketcheson 1999). Para esse tipo de teste, é necessário que as amostras de água tenham salinidade mínima de $20 \mathrm{~g} / \mathrm{l}$. No caso da água usada na simulação, a salinidade chega a $35 \mathrm{~g} / 1$ por amostra. O analisador usado nesse teste é o Microtox, modelo 500, fabricado pela Microbics.

Análise dos Nutrientes Os nutrientes dissolvidos na água usada durante a simulação, são analisados num espectrofotômetro, marca Schimadzu, modelo UV-160 A. 

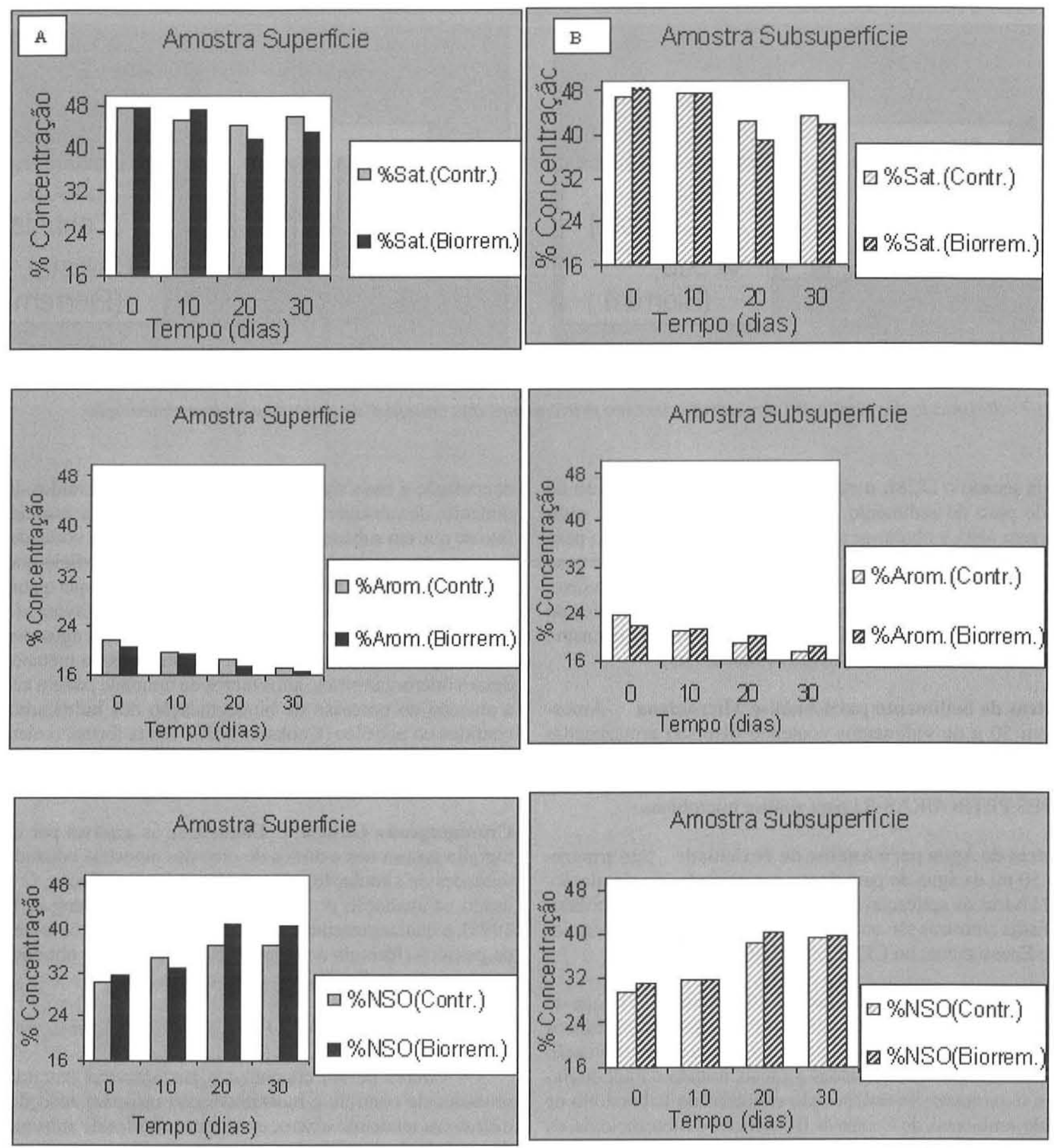

Figura 6-Análise por cromatografia líquida dos extratos de amostras de óleo provenientes das unidades de simulação: (A) amostras de superficie; (B) amostras de subsuperficie.

A análise do fosfato inorgânico é iniciada com a preparação de solução padrão contendo ortofosfato, com subseqüente diluição seqüencial. Posteriormente, são preparados alguns reagentes, tais como: reagente 1 (molibdato de amônio, tartarato de antimônio e potássio); reagente 2 (ácido ascórbico); reagente 3 (mistura de ambos reagentes). As amostras são dispostas em copos plásticos, e posteriormente são analisadas no espectrofotômetro, para a leitura do comprimento de onda de $885 \mathrm{~nm}$.

Quanto a análise do íon amônia é iniciada com a preparação de solução padrão de cloreto de amônio, seguida de diluição seqüencial. São preparados determinados reagentes: reagente 1 (fenol, etanol, nitroprussiato de potássio); reagente 2 (citrato de sódio, não); reagente 3 (trione); reagente 4 (mistura de reagentes). Posteriormente as amostras são dispostas em copos plásticos, com adição de padrões e água deionizada e injetadas no espectrofotômetro, com comprimento de onda de $630 \mathrm{~nm}$.

\section{AMOSTRAGEM AO LONGO DA SIMULAÇÃO}

Amostras de Sedimento contendo Óleo para Cromatografia As amostras contendo óleo são retiradas de cada unidade de simulação, em duas profundidades distintas, representando a superfície (até $2 \mathrm{~cm}$ ) e subsuperfície (entre 2 e $5 \mathrm{~cm}$ ). A extração do óleo contido nos sedimentos envolve: transferência de $10 \mathrm{~g}$ de amostras de sedimentos contendo óleo para frascos com capacidade de $250 \mathrm{ml}$; adição de $30 \mathrm{ml}$ de diclorometano (DCM) com agitação manual durante 10 minutos; extração do DCM da solução através de evaporador rotativo, à temperatura de $40^{\circ} \mathrm{C}$; transferência do concentrado para o vial, levado à capela para 

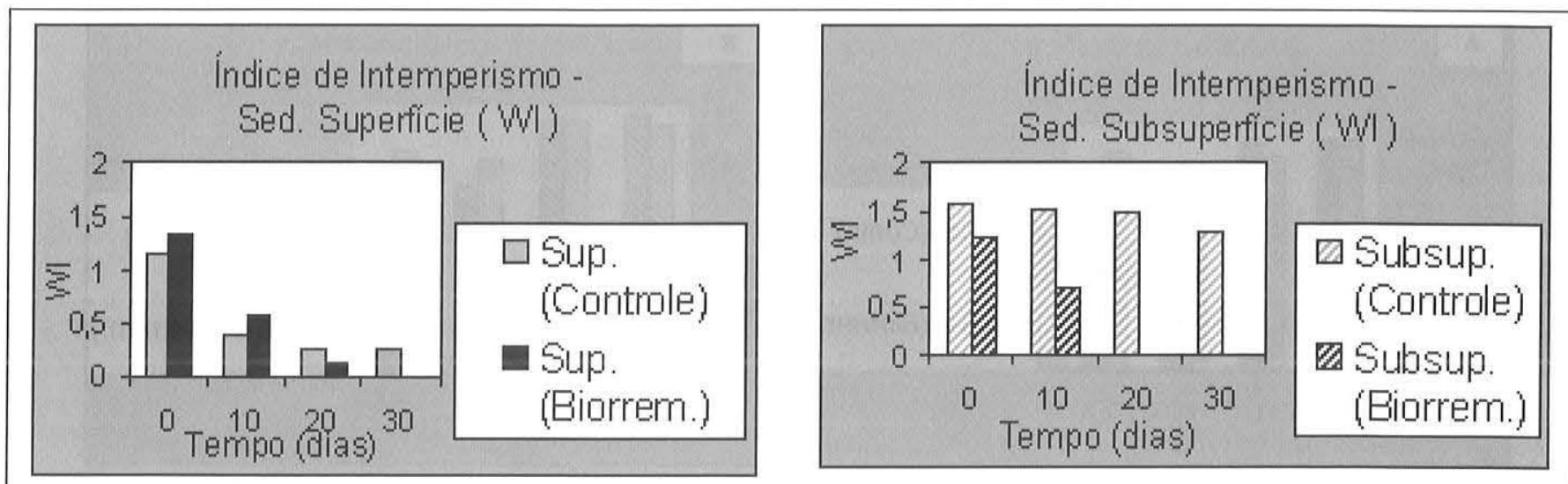

Figura 7-Resultado das razões WI dos extratos de óleo provenientes das unidades de controle e de biorremediação.

que seja secado o DCM; o vial é pesado, obtendo-se o peso do óleo; do peso do sedimento inicial subtrai-se o peso do sedimento sem óleo, e obtém-se assim o peso do sedimento; o peso da água contido nas amostras é determinado subtraindo do peso inicial da amostra $(10 \mathrm{~g})$, o peso do sedimento e o peso do óleo. Essa etapa da análise é realizada no Laboratório de Química do Centro Federal de Educação Tecnológica, na Unidade de Ensino Descentralizada (CEFET/UNED), em Macaé (RJ).

Amostras de Sedimento para Análise Microbiana Amostras com $50 \mathrm{~g}$ de sedimentos contendo óleo são armazenadas em frascos de polietileno e mantidas refrigeradas até encaminhamento à Gerência de Biotecnologia e Ecossistemas do CENPES/PETROBRAS-RJ para análise microbiana.

Amostras de Água para Análise de Toxicidade São armazenados $50 \mathrm{ml}$ da água de percolação das unidades de simulação, após 24 horas da aplicação de fertilizante, em frascos de polietileno. Essas amostras são encaminhadas á Gerência de Biotecnologia e Ecossistema, no CENPES/PETROBRAS-(RJ).

Amostras de Água para Análise dos Nutrientes A água do mar extraída das colunas de simulação é armazenada em frascos de polietileno, com capacidade de $40 \mathrm{ml}$. Antes da determinação do teor de nutrientes, são filtradas a vácuo, usando o funil de $B u$ chner e o quitazato. As análises são efetuadas no Laboratório de Controle Ambiental, do Centro de Biociências e Biotecnologia, da Universidade Estadual do Norte Fluminense (LCA/CBB/UENF).

\section{RESULTADOS E DISCUSSÂO}

Cromatografia Líquida Efetuam-se análises por cromatografia líquida dos extratos de óleo extraídos das amostras dos sedimentos provenientes das unidades de controle e de biorremediação, nos sedimentos de superfície e subsuperfície.

Nas análises dos óleos extraídos de todas unidades de simulação, exibidos na Fig.6, os resultados mostram uma redução na concentração relativa da fração dos hidrocarbonetos saturados, seguida de uma redução relativa e menos expressiva dos aromáticos, compensada pelo aumento relativo na fração dos compostos polares (NSO). Esses dados comprovam a ordem da escala de biodegradação proposta por alguns autores, como McMillen et al. (1993), em que os saturados são os hidrocarbonetos mais suscetíveis à degradação microbiana, quando comparados aos outros compostos contidos no óleo.

A comparação dos resultados das amostras de óleo extraídas dos sedimentos de superfície com as amostras dos sedimentos de subsuperfíce, das unidades de simulação, mostram que a bio- degradação é mais significativa nas dos óleos extraídos dos sedimentos de subsuperfície. Tal resultado pode estar associado ao fato de que em subsuperfície se mantêm teores de umidade altos e constantes, ao contrário das amostras de superfície que perdem água nas fases intermarés. Considerando-se que o aumento da quantidade de água disponível no ambiente favorece o desenvolvimento da população microbiana, e que a água disponível é responsável pelo transporte de nutrientes no metabolismo desses microrganismos, altos teores de umidade podem acelerar a atuação do processo de biodegradação dos hidrocarbonetos contidos no petróleo (Cookson 1995). Desta forma, o óleo contido nos sedimentos de subsuperfície pode ser mais suscetível a biodegradação do que o contido nos sedimentos de superfície.

Cromatografia Gasosa Efetuam-se as análises por cromatografia gasosa nos extratos de óleo das amostras coletadas das unidades de simulação de controle e biorremediação. O índice usado na avaliação do intemperismo é o WI (Wang \& Fingas 1994), o qual representa a razão dos hidrocarbonetos leves sobre os pesados (fórmula a seguir) e cujos resultados obtidos estão apresentados na Fig. 7.

$$
(\mathrm{WI})=\left(n-\mathrm{C}_{8}+n-\mathrm{C}_{10}+n-\mathrm{C}_{12}+n-\mathrm{C}_{14}\right) /\left(n-\mathrm{C}_{22}+n-\mathrm{C}_{24}+n-\mathrm{C}_{26}+n-\mathrm{C}_{28}\right)
$$

Os valores de WI encontrados nas amostras extraídas das unidades de controle e biorremediação mostram reduções significativas tendendo a zero, exceto na amostra de subsuperfície da unidade de controle. Este resultado é plausível considerando que, dentre os processos intempéricos, a evaporação é o mais atuante em todas as unidades de simulação, sendo responsável pela redução dos hidrocarbonetos de baixo peso molecular (Wang \& Fingas 1995). Em subsuperfície, a aplicação de nutrientes favorece a atuação do processo de biorremediação.

$\mathrm{Na}$ análise do processo de biodegradação, utilizam-se os índices $n-\mathrm{C}_{17} /$ pristano, $n$ - $\mathrm{C}_{18} /$ fitano e pristano/fitano (Fig. 8), os quais mostram uma redução nos seus valores nos óleos extraídos dos sedimentos de superfície e subsuperfície. Entretanto, nas amostras de subsuperfície essa redução é mais acentuada, havendo uma queda brusca a partir de dez dias e reduzindo a zero já em vinte dias do experimento. Nas amostras de superfície esse índice só foi reduzido a zero a partir de 30 dias, comprovando que a atuação da biodegradação é mais rápida nos óleos contidos nas amostras de sedimentos de subsuperfície do que nas de superfície.

A redução das razões $n \mathrm{C}_{17} / \mathrm{p}$ e $n \mathrm{C}_{18} / \mathrm{f}$, obtidas nas unidades submetidas a biorremediação, corroboram a ordem crescente de susceptibilidade dos hidrocarbonetos ao ataque microbia- 

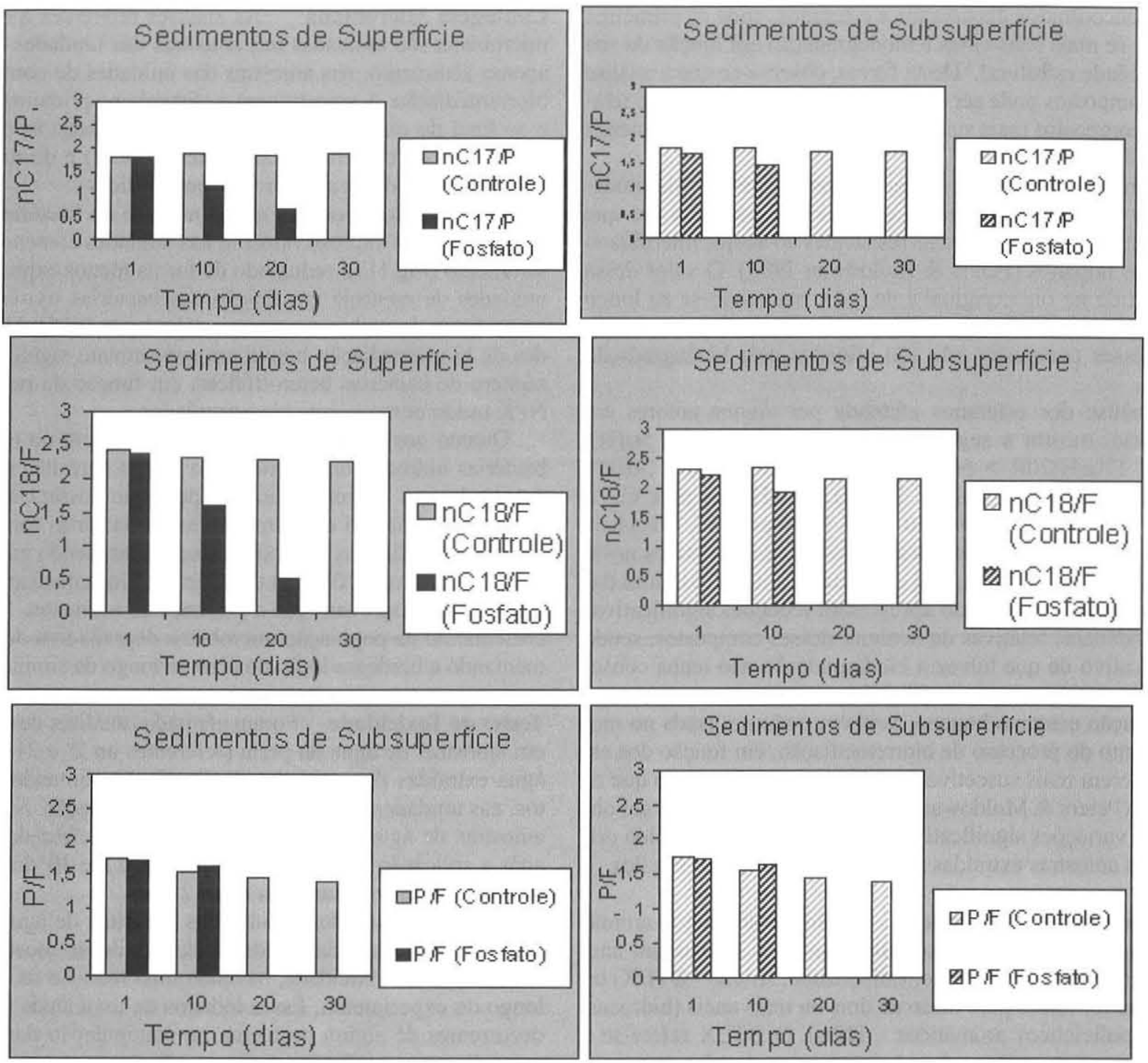

Figura 8 - Resultado das razões $n C_{1}$, pristano, $n C_{1}$, fitano e pristano/fitano dos extratos de amostras de óleo proveniente das unidades de simulação de controle e de biorremediação, nos sedimentos de (A) superficie e (B) subsuperficie.

no proposto por Brown (1987), Bradford \& Krishnomoorthy (1991), e Peters \& Moldowan (1993) onde os alcanos lineares são mais susceptíveis a biodegradação que os alcanos ramificados. Segundo experimentos efetuados por Restle (1983), a biodegradação mostra-se mais eficiente nas $n$-parafinas, principalmente de tamanhos menores, seguidas das isoparafinas e de hidrocarbonetos aromáticos.

A diminuição das razões dos biomarcadores pristano/fitano em todas as amostras analisadas indica o avanço da biodegradação. As amostras de óleo extraídas das unidades de controle mostram valores altos e constantes para esses índices, e as amostras de óleo provenientes das unidades de simulação de biorremediação mostram uma redução significativa, comprovando que a aplicação de nutrientes é efetiva na aceleração do processo de biodegradação, inclusive dos isoprenóides, preferencialmente do pristano.

$\mathrm{Na}$ análise da atuação do processo de biodegradação, além dos índices de biodegradação anteriormente descritos, verificase a presença de compostos não resolvidos cromatograficamente, identificados por um hump na linha de base dos fingerprints.

Cromatografia Gasosa Acoplada à Espectrometria de Massas (CG/EM) Considerando que os óleos constituem-se de diferentes compostos químicos e esses apresentam uma ordem de degradação definida por alguns autores, em função da sua complexidade estrutural, a identificação e quantificação detalhada desses compostos contribuem na análise do grau de biodegradação desse óleo. Segundo Peters \& Moldowan (1993), os biomarcadores cíclicos são moléculas que apresentam um grau de estabilidade química maior que os $n$-alcanos lineares e isoalcanos frente ao ataque microbiano. Entretanto, com a redução completa desses compostos e com o aumento da atuação do processo de biodegradação, os biomarcadores cíclicos passam a ser degradados e suas concentrações tornam-se reduzidas. Através da cromatografia gasosa acoplada a espectrometria de massa, pode-se analisar detalhadamente a fração dos hidrocarbonetos saturados e dos aromáticos contidos no óleo.

Hidrocarbonetos saturados dentre os biomarcadores cícli$\cos$ dos hidrocarbonetos saturados alguns são mais suscetiveis a biodegradação, destacando-se os terpanos (fragmentogramas $\mathrm{m} / \mathrm{z} 191$ ) e esteranos (fragmentogramas $\mathrm{m} / \mathrm{z} 217$ ).

Nos fragmentogramas m/z 191 tanto o óleo original como as amostras das unidades de simulação apresentam: terpanos tricíclicos (19 a 23 átomos de carbono), terpanos tetracíclicos (24 a 28 átomos de carbono), e terpanos pentacíclicos ou hopanos (28 a 35 átomos de carbono). Já nos fragmentogramas $\mathrm{m} / \mathrm{z}$ 
217 são encontrados diasteranos e esteranos, onde os primeiros mostram-se mais resistentes a biodegradação em função da sua complexidade estrutural. Desta forma, observa-se que a análise desses compostos pode ser efetuada através de índices que relacionam compostos mais suscetíveis a biodegradação dos menos biodegradáveis.

Dentre as razões mais usadas no monitoramento da biodegradação encontra-se a razão tricíclicos/hopanos, uma vez que os terpanos tricíclicos são mais resistentes ao ataque microbiano do que os hopanos (Peters \& Moldowan 1993). O valor dessa razão obtido no óleo original é de 0,43 , mantendo-se ao longo dos experimentos em todas unidades de simulação, o que indica que esses compostos não são afetados pela biodegradação (Fig.9a).

A análise dos esteranos efetuada por alguns autores em laboratório, mostra a seguinte ordem de degradação: $5 \alpha(\mathrm{H})$, $14 \alpha(\mathrm{H}), 17 \alpha(\mathrm{H}) 20 \mathrm{R}>5 \alpha(\mathrm{H}), 14 \alpha(\mathrm{H}), 17 \alpha(\mathrm{H}) 20 \mathrm{~S}>5 \alpha(\mathrm{H})$, $14 \beta(\mathrm{H}), 17 \beta(\mathrm{H}) 20 \mathrm{R}>5 \alpha(\mathrm{H}), 14 \beta(\mathrm{H}), 17 \beta(\mathrm{H}) 20 \mathrm{~S}$ para $\mathrm{C}_{27}>$ $\mathrm{C}_{28}>\mathrm{C}_{29}$ (Seifert \& Moldowan 1979, Connan 1984, Macknzie \& McKenzies 1983). Os resultados dessas razões obtidos nesse trabalho, tanto no óleo original como nas amostras extraídas das unidades de simulação, não apresentam reduções significativas nas abundâncias relativas de nenhum desses compostos, sendo um indicativo de que talvez a biodegradação não tenha conseguido atingir tais compostos (Fig.9b).

A relação esterano/hopano, também pode ser usada no monitoramento do processo de biorremediação, em função dos esteranos serem mais suscetíveis ao ataque microbiano do que os hopanos (Peters \& Moldowan 1993). Entretanto, não foram observadas variações significativas dessas razões entre o óleo original e as amostras extraídas ao longo da simulação (Fig.9c).

Hidrocarbonetos aromáticos Os hidrocarbonetos aromáticos são constituídos de moléculas contendo apenas um anel benzênico (Benzeno, Tolueno, Etilbenzeno, Xileno - BTEX) ou moléculas formadas pela fusão de dois ou mais anéis (hidrocarbonetos policíclicos aromáticos - HPA). O BTEX refere-se a compostos muito voláteis e de baixo peso molecular, enquanto que os HPA são moléculas maiores que podem ser recalcitrantes no sítio de contaminação e menos suscetíveis a biodegradação quando comparados aos outros compostos orgânicos (Barker 1980, Connan 1984). A ordem de degradação dos HPA respeita o tamanho da molécula, ou seja, quanto menor for o composto mais suscetível este será ao ataque microbiano. A presença de ramificações e de heteroátomos (NSO) também dificultam a biodegradação (Rubinstein et al. 1977, Deroo et al. 1977, Connan 1984, Wilson \& Jones 1993).

Alguns autores (Wardroper et al. 1984, Rowland et al. 1986, Wang \& Fingas 1995, Fayad \& Overton 1995), através de resultados obtidos em experimentos laboratoriais, propuseram a seguinte ordem de degradação para os HPA: Naftaleno $>$ Fluoreno $>$ Dibenzotiofeno $>$ Fenantreno $>$ Criseno.

Com o objetivo de avaliar a atuação da biorremediação nos HPA da amostra de óleo original e das amostras extraídas das unidades desimulação, foram monitorados os seguintesíons(Fig.10): $156 \mathrm{C} 2$-naftaleno (1,6dimetilnaftaleno/2,6dimetilnaftaleno), 170 C3-naftaleno (1,3,5trimetilnaftaleno+1,4,6trimetilnaftaleno/ 1,2,6trimetilnaftaleno), 180 C1-fluoreno (2metilfluoreno/ 1metilfluoreno), 192 C1-fenantreno (3metilfenantreno/ 2metilfenantreno) e $198 \quad$ C1-dibenzotiofeno (2,3metildibenzotiofeno/1metildibenzotiofeno).

Da mesma forma que nos resultados anteriormente descritos dos hidrocarbonetos saturados, os resultados dos hidrocarbonetos aromáticos não mostram reduções significativas nas suas abundâncias relativas ao longo da simulação, indicando a ausência da atuação da biodegradação nesses compostos durante a simulação.
Contagem Microbiana As análises referentes à contagem microbiana são efetuadas nas amostras das unidades contendo apenas sedimento, nas amostras das unidades de controle e de biorremediação. A amostragem é efetuada no primeiro dia (1 d) e ao final do experimento (30 d). São realizadas as contagens do número de bactérias totais (heterotróficas) e do número de bactérias degradadoras (hidrocarbonoclásticas).

Os resultados mostram que o número de bactérias heterotróficas reduziu expressivamente nas unidades contendo apenas sedimento (Fig.11) e reduzindo de forma menos expressiva nas unidades de controle em função das bactérias usarem o óleo como fonte de carbono e energia (Cookson 1995). Nas unidades de biorremediação houve um crescimento significativo no número de bactérias heterotróficas, em função da presença do NPK usado como agente bioestimulador.

Quanto aos resultados obtidos na contagem do número de bactérias hidrocarbonoclásticas, no tempo zero não é possível detecta-las em número suficiente para que possam ser quantificadas. Ao final do experimento, essas bactérias foram detectadas e quantificadas em todas as unidades, sendo encontradas em maiores proporções nas unidades de biorremediação. Esses resultados comprovam que a presença de nutrientes favorece o crescimento da população microbiana degradadora de óleo, aumentando a biodegradação do óleo ao longo da simulação.

Testes de Toxicidade Foram efetuadas análises de toxicidade em amostras de água da praia (referentes ao $2^{\circ}$ e $21^{\circ}$ dia) e de água extraídas das unidades de simulação contendo sedimentos, nas unidades de controle e de biorremediação. A coleta das amostras de água ao longo da simulação é efetuada 24 horas após a aplicação de fertilizantes $\left(2^{\circ}, 11^{\circ}, 21^{\circ}\right.$ e $30^{\circ}$ dia $)$, e os resultados são apresentados na Fig. 12.

Tanto os resultados obtidos das amostras de água da praia como das amostras das unidades de simulação mostra na fase inicial sinal de toxicidade, havendo uma redução da mesma ao longo do experimento. Esses indícios de toxicidade podem ser decorrentes de algum problema na manipulação das amostras antes de serem analisadas, portanto não devem ser considerados com cautela ou mesmo desconsiderados.

Todavia, ao longo do experimento, tais indícios de toxicidade mostraram-se reduzidos e as respostas apresentaram-se semelhantes em todas as unidades, o que comprovou que ao longo da simulação não houve um efeito tóxico do fertilizante sobre a bactéria Vibrio fischeri.

Concentrações de Nutrientes Efetuam-se análises dos teores dos íons de amônio e fosfato em amostras de água extraídas das unidades de simulação contendo apenas sedimento, das unidades de controle e de biorremediação. A retirada das amostras de água é efetuada 24 horas após a aplicação de fertilizante. Os resultados são mostrados na Fig. 13.

As análises dos resultados dos teores do íon amônio e íon fosfato mostram comportamentos semelhantes. Nas unidades de controle, as concentrações desses nutrientes mantêm-se baixas e constantes, e nas unidades de biorremediação há um aumento significativo nos teores desses compostos, atingindo valores máximos em torno de 21 dias, reduzindo-se ao final dos experimentos em função das suas elevadas solubilidades em água.

$\mathrm{O}$ aumento nos teores de nutrientes favorece o crescimento da população microbiana responsável pela biodegradação do óleo contido no ambiente. Os resultados encontrados corroboram a idéia de alguns autores de que em sistemas abertos pode haver deficiência nos teores de nutrientes (Lindstrom et al. 1991, Furnas 1992 apud Hoff 1993, Bragg 1992 apud Rytkonen et al. 1997), resultando na inibição da atuação do processo de biodegradação, o qual pode ser favorecido pela adição de ferti- 


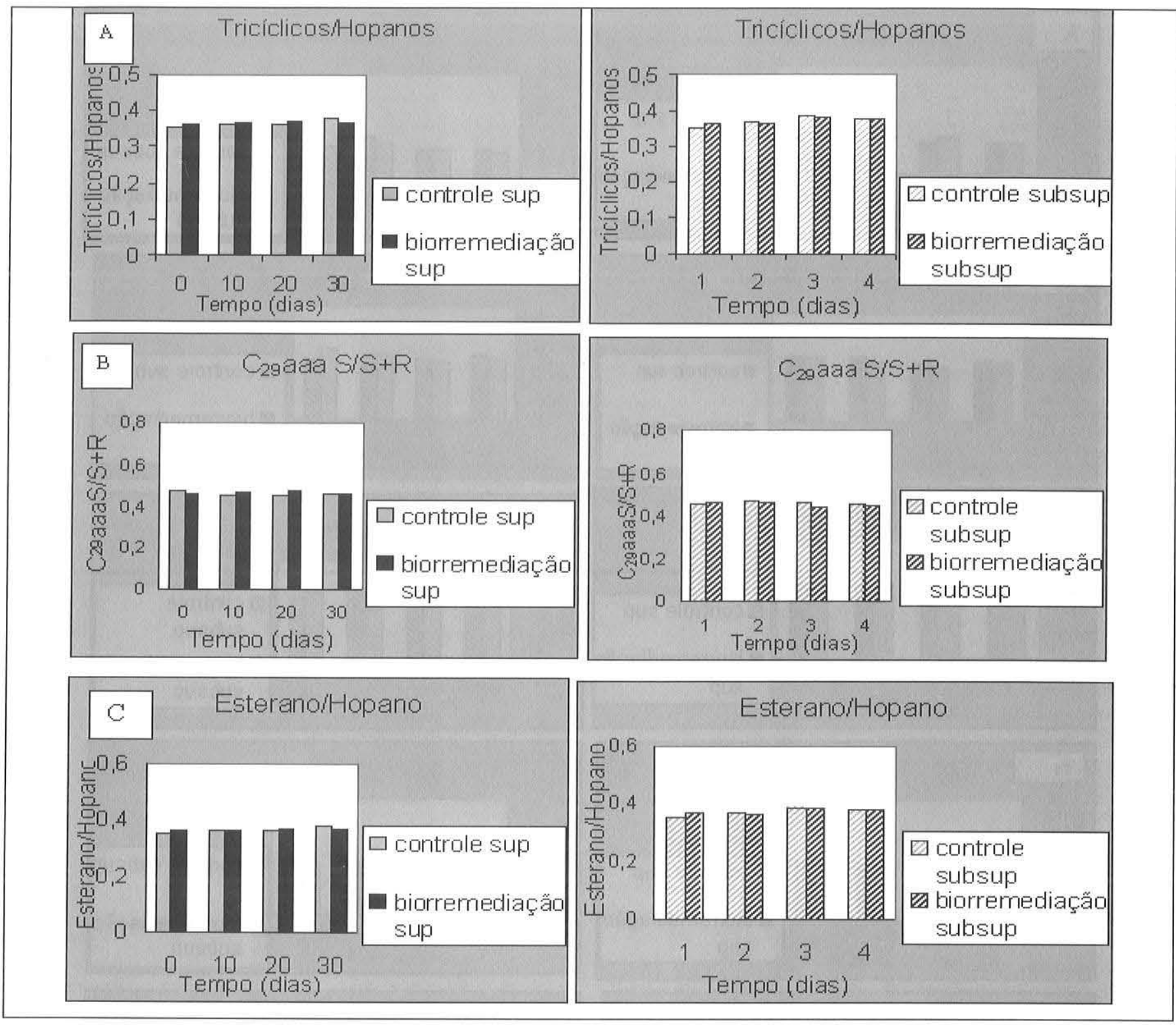

Figura 9-Resultado da análise por CG/EM dos extratos de óleo retirados das amostras das unidades de simulação dos sedimentos de superficie (esquerda) e subsuperficie (direita), mostrando algumas razões usadas como indice de biorremedição: (A) triciclicos/hopanos, (B)C29aaa $S / S+R$, (C) esteranos/hopanos.

lizantes ao ambiente.

Extrapolando os resultados para o ambiente real, deve-se considerar ainda que os sistemas costeiros como praias, referese à ambientes abertos, ou seja, diariamente a maré responsabiliza-se pela lavagem dos sedimentos encontrados nesses locais. Assim, para o cálculo de quantidade de nutrientes em ambientes costeiros impactados deve-se levar em consideração a energia dos ambientes.

CONCLUSÕES A interpretação dos resultados obtidos pelo presente trabalho atinge o objetivo proposto inicialmente, que é avaliar a aplicabilidade da técnica de biorremediação, através da bioestimulação pelo nutriente NPK, na mitigação de impactos ambientais costeiros gerados por derrames de óleo. Dentre os principais resultados destacam-se:

- Houve uma redução relativa na fração dos hidrocarbonetos saturados, compensado pelo enriquecimento relativo em compostos polares (NSO), mantendo-se constante a fração dos hidrocarbonetos aromáticos durante o processo de biorremediação;

- Ocorre uma redução maior dos alcanos lineares, quando comparado à dos isoprenóides, corroborando a idéia de susceptibilidade de biodegradação;

- As amostras de óleo extraídas da subsuperfície das unidades de simulação mostram uma maior atuação da biodegradação do que às extraídas da superfície decorrente dos maiores teores de umidade contidos nesse local;

- Não são observadas variações nas abundâncias relativas dos compostos saturados terpanos tricíclicos, pentacíclicos (hopanos) e esteranos contidos no petróleo, nem dos hidrocarbonetos policíclicos aromáticos naftaleno, fluoreno, fenantreno e dibenzotiofeno.

- O fertilizante não mostra nenhum efeito tóxico sobre a biota local, não comprometendo assim a aplicabilidade da técnica; 

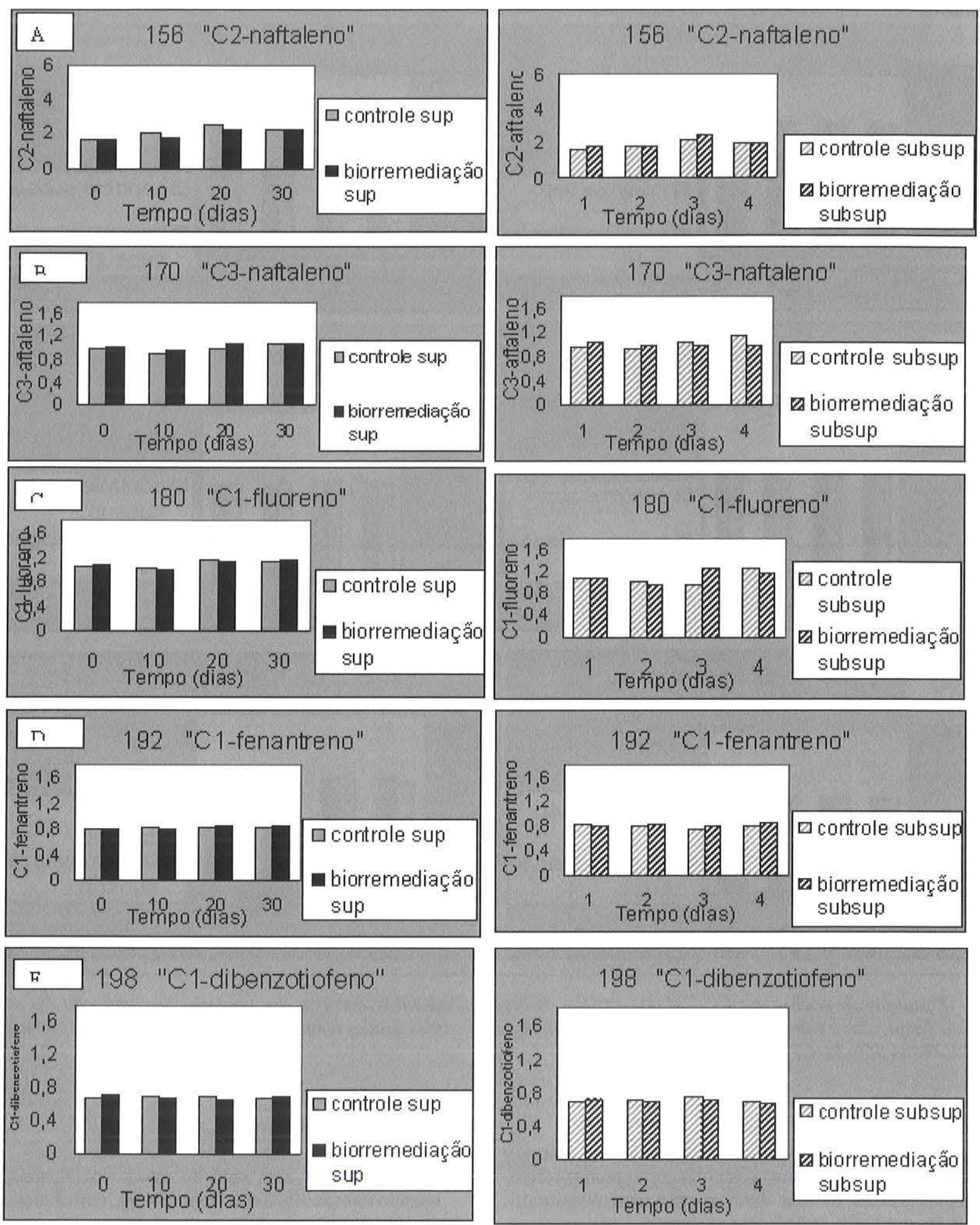

Figura 10 - Razões dos íons 156 (C2-naftaleno), 170 (C3-naftaleno) 180 (C1-fluoreno), 192 (C1-fenantreno), 198 (C1-dibenzotiofeno) dos extratos de óleo retirado da amostras de superficie (direta) e subsuperficie (esquerda) das unidades de controle e biorremediação.

- Não é verificada a saturação de nutrientes no ambiente, proveniente da aplicação de fertilizante, considerando que o ambiente costeiro é um sistema aberto e lavado diariamente pela maré;

Concluímos que, ao final da simulação, há uma degradação completa dos $n$-alcanos e uma degradação parcial dos isoprenóides, sendo mantida inalterada as abundâncias relativas dos biomarcadores hopanos e esteranos. Desta forma, a biorremediação pode ser classificada como moderada, atingindo o nível cinco na escala de classificação feita por Peters \& Moldowan (1993).

Agradecimentos À FINEP/CTPETRO pelo financiamento a este projeto. Os autores agradecem a Dra. Antônia Volpon e Dra. Silvana Barbante do CENPES/PETROBRAS - pelo auxílio no desenvolvimento desse projeto e pelas sugestões técnicas propostas. Ao CENPES/PETROBRAS pelo apoio ao desenvolvimento desta pesquisa. 


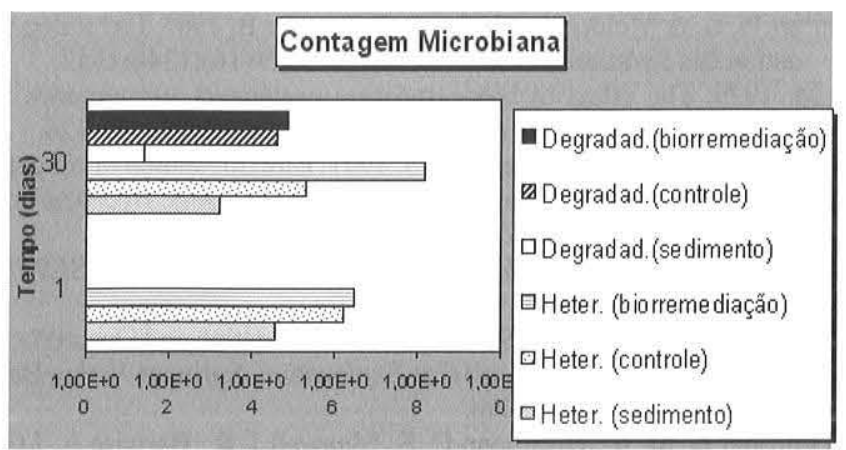

Figura 11 - Resultado da contagem microbiana efetuada nas amostras retiradas das unidades de simulação contendo apenas sedimento, de controle e de biorremediação.

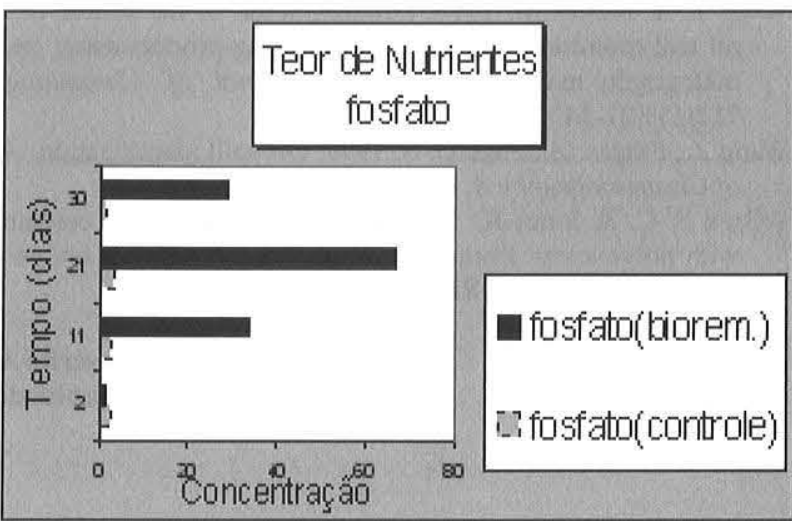

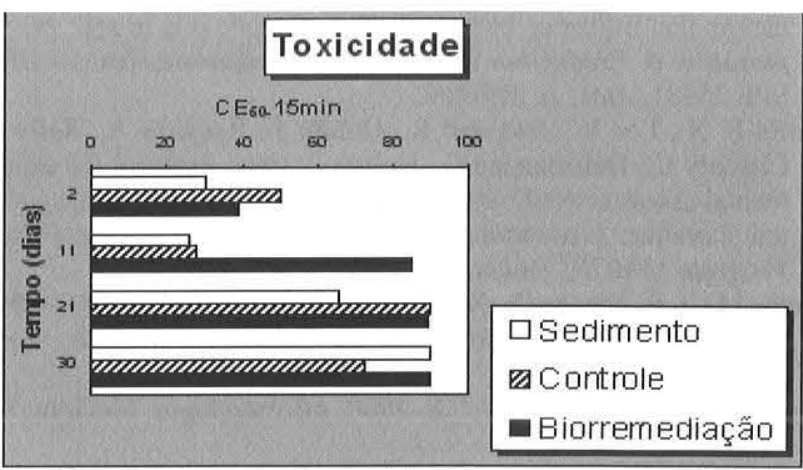

Figura 12 - Resultado dos testes de toxicidade efetuada nas amostras de água extraída das unidades de simulação contendo sedimento, das unidades de controle e de biorremediação.

Figura 13 - Resultados das análises dos teores de nutrientes das amostras de água provenientes das unidades de controle e biorremediação.

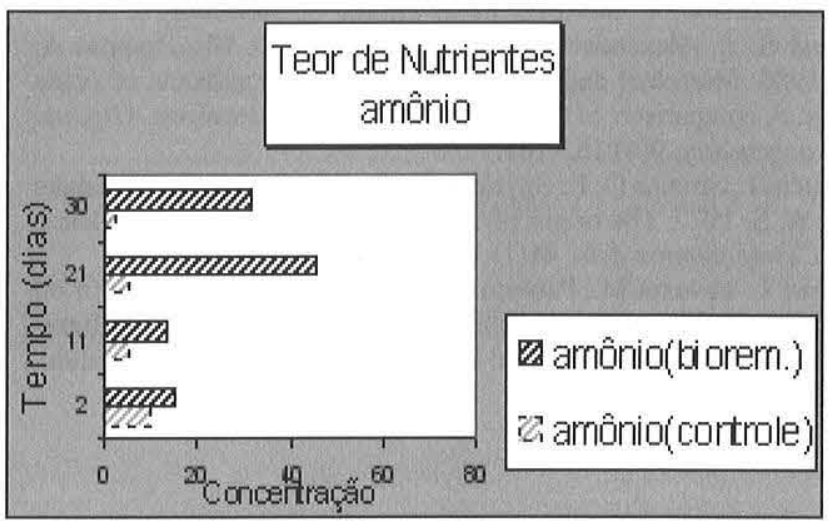

\section{Referências}

Alexander M.1981. Biodegradation of chemicals of environmental concern. Science, 2(11):132-138.

Al-Hadhrami M. N., Lappin-Scott H. M., Fisher P. J. 1997. Studies on the biodegradation of three groups of $n$-alcanes in the presence of molasses and mineral fertilizer by Pseudomonas aeruginosa. Marine Pollution Bulletin, 34(11): 969-974.

Atlas R. M. 1981. Microbial degradation of petroleum hydrocarbons: an environmental perspective. Microbiology, 45(1):180-209.

Barker C. 1980. Application of geochemistry in exploration. Maidenhead, 28July.

Bartha R. 1996. Biotechnology of petroleum pollutant biodegradation. Microbial Ecology, 12(1):155-172.

Bragg J. R. 1992. Bioremediation for shoreline cleanup following the 1989. Alaskan Oil Spill, In: Exxon Research \& Engineering Co., 1$94 \mathrm{p}$.

Bradford M. L. \& Krishnamoorthy R. 1991. Consider bioremediation for waste site cleanup. Chemical Engineering Progress, 87(2):8085.

Brown L. R. 1987. Oil-degrading microrganisms. Chemical Engineering Progress, 1(1):35-40.

Chartre S., Purohit H., Shanker R., Khanna P. 1996. Bacterial consortia for crude oil spill remediation. Wat. Sci. Tech., 34(10):187-193.

Connan J. 1984. Biodegradation of crude oils ins reservoirs. In: Brooks, J. \& Welte, D. H. (Eds.) Advances in Petroleum Geochemistry. Academy Press, NY, Vol.1, p.:299-335.

Cookson T. 1995. Bioremediation Engineering: Design and Application. McGraw-Hill Inc, New York, 525p.
Crapez M. A. C. 1982. Isolement à Partir du Sol et Bactéries Aérobies Sporullées Dégradant Composés Aromatiques. Tese de Doutoramento. Université D'Aix-Marseille II, França, p. 1-76.

Del 'Arco J. P. 1999. Degradação de Hidrocarbonetos por Bactérias e Fungos em Sedimento Arenoso. Tese de Doutoramento. Instituto de Química, Universidade Federal do Rio de Janeiro-UFRJ, 169p.

Deroo G., Powell T. G., Tissot B., McGrossan R. G. 1977. Geological Survey. Canadian Bulletin, 1(1):231-262.

Fayad N. M. \& Overton E. (1995) A unique biodegradation pattern of the oil spill during the 1991 Gulf War. Marine Pollution Bulletin, 30(4):239-246.

Furnas M. J. 1992. The behavior of nutrients in tropical aquatic ecosystems. Pollution in Tropical Aquatic Systems, 1(1):30-65.

Hoff R. Z. 1993. Bioremediation: an overview of its development and use for oil spill cleanup. Marine Pollution Bulletin, 26(9):476481.

Ketcheson K. 1999. Microtox Acute. Toxicity Procedure. Emergencies Science Division. Ottawa, Ontario. $21 \mathrm{p}$.

Lindstrom J. E., Prince R. C., Clark R. C., Grossman J. C., Yeager T. R., Braddock J. F., Brown E. J. 1991. Microbial populations and hydrocarbon biodegradation potentials in fertilized shoreline sediments affected by the T/V Exxon Valdez oil spill. Appli. Env. Micro, 57(1): 2514-2522.

Mackenzie A. S. \& McKenzies D. 1983. Isomerization and aromatization of hydrocarbons in sedimentary basins formed by extension. Geology Maganize, 120(1):417-470.

McMillen S. J., Kerr J. M., Gray N. R. 1993. Microcosm studies of 
factors that influence bioremediation of crude oils in soil. In: $E x$ ploration \& Production Environments Conference, Texas - USA, SPE 25981, Atas, p. 389-400.

Merlin F. X., Lee K., Swannel R., Outdot J., Basseres A., Relly T., Chauery C., Dalmazzone C., Sveum P. 1994. Protocol for experimental assessment of bioremediation agents on a petroleum polluted shoreline. Proceeding of the 17th Arctic and Marine Oilspill Program (AMOP) Technical Seminar, 1:465-478.

Mitchell G. I. P., Muche D., Ximenez M. S., Harris S., Nassar C. 1993. Programa Ambiental da Bacia de Campos. Petrobrás, Vol.1, $169 \mathrm{p}$.

Pelczer M., Reid R., Chan E.C.S. 1980. Microbiologia. McGraw-Hill do Brasil, 1:129-147.

Peters K. E. \& Moldowan J. M. 1993. The Biomarker Guide: Interpreting Molecular Fossil in Petroleum and Ancient Sediments. Vol.1, Prentice Hall Press, N. Jersey, 361p.

Prince R. C. 1993. Petroleum spill bioremediation in marine environments. Microbiology, 19(4):217-242.

Restle A. 1983. Eude de nouveaux marqueurs biologiques dasns dês petroles biodegrades: cas naturels e. simulations in vitro. Tese de Doutoramento. L'universite Louis Pasteur de Strasbourg I, 218p.

Rowland S. J., Alexander R., Kagi R. I., Jones D. M., Douglas A. G.1986. Microbial degradation of aromatic components of crude oils: A comparison of laboratory and field observations. Organic Geochemistry, 9(4):153-161.

Rubinstein I., Strausz O. P., Spyckerelle C., Crawford R. J., Westalake D. W. S. 1977. The origin of oil bitumens of Alberta. Geochimica et Cosmochimica Acta. 41(1):1341-1353.

Rytkonen J., Itavaara M., Paulsen J. E. 1997. The Improvement of oil biodegradation on the shoreline. In: Proceedings of the 7th International Offshore and Polar Engineering Conference, Honolulu, USA, 2(1):25-30.
Seifert W. K. \& Moldowan Slivins A. \& Tremier B. 1985. La biodégradation des hydrocarbures. La Recherché, 171(16):1344-1352.

J. M. 1979. The effect of biodegradation onsteranes and terpanes in crude oils. Geochemica et Cosmochimica Acta, 43(1):111-126.

Sveum P., Faksness L. G., Ramstad S. 1991. Bioremediation of oil-contaminated shorelines: the role of carbon in fertilizers. Hydrocarbon Bioremediation, 1(2): 163-174.

Teixeira Neto A. S. 1980. Manual de Sedimentologia. DIVEN/SEPES/ Petrobrás, Bahía, Vol.1, 194p.

Tissot B. P. \& Welte D. H. 1978. Petroleum Formation and Occurrence. A New Approach to Oil and Gas Exploration. Springer-Verlag Berlin Heidelberg, Vol.1, 540p.

Wardroper A. M. K., Hoffmann C. F., Maxwell J. R., Barwise A. J.G., Goodwin N. S., Park P. J. D. 1984. Crude oil biodegradation under simulated and natural conditions - II. Aromatic steroid hydrocarbons. Organic Geochemistry, 6(1): 605-617.

Wang Z. \& Fingas M. 1994. Study of the effects of weathering on the chemical composition of light crude oil. In: Proceeding of the 17th Arctic and Marine Oilspill Program (AMOP) Technical Seminar, 1(1):133-171.

Wang Z. \& Fingas M. 1995. Differentiation of the source of spilled oil and monitoring of the oil weathering process using gas chromatography-mass spectrometry. Journal of Chromatography, 712(1):321-343

Wang Z., Fingas M., Page D. S. 1999. Oil spill identification. Journal of Chromatography A, 843(1):369-411.

Wilson S. C. \& Jones K. 1993. Bioremediation of soil contaminated with polynuclear aromatic hydrocarbons (PAHs): a review. Environmental Pollution, 81(1)229-249.

Manuscrito A-1564 Revisão aceita em 14 de junho de 2006 